\title{
A review of endovascular treatment for medium vessel occlusion stroke
}

\author{
Johanna Maria Ospel (i) 1,2 Mayank Goyal (1) 2,3
}

\begin{abstract}
- Additional material is published online only. To view, please visit the journal online (http://dx.doi.org/10.1136/ neurintsurg-2021-017321).

${ }^{1}$ Radiology, Universitatsspital Basel, Basel, Switzerland ${ }^{2}$ Clinical Neuroscienes, University of Calgary, Calgary, Alberta, Canada

${ }^{3}$ Diagnostic Imaging, University of Calgary, Calgary, Alberta, Canada
\end{abstract}

Correspondence to Dr Mayank Goyal, Diagnostic Imaging, University of Calgary, Calgary, AB T2N 1N4, Canada; mgoyal2412@gmail.com

Received 15 January 2021 Revised 17 February 2021 Accepted 17 February 2021 Published Online First 26 February 2021

\section{Check for updates}

(C) Author(s) (or their employer(s)) 2021. No commercial re-use. See rights and permissions. Published by BMJ.

To cite: Ospel JM, Goyal M. J Neurolntervent Surg

2021:13:623-630

\begin{abstract}
Medium-vessel occlusions (MeVOs), that is, occlusions of the $\mathrm{M} 2 / 3$ middle cerebral artery, $\mathrm{A} 2 / 3$ anterior cerebral artery, and $\mathrm{P} 2 / 3$ posterior cerebral artery segments, account for $25 \%-40 \%$ of all acute ischemic stroke cases. Clinical outcomes of MeVO stroke with intravenous thrombolysis, which is the current standard of care, are moderate at best. With improving imaging technologies and a growing literature, MeVOs are increasingly recognized as a target for endovascular treatment (EVT). For the time being, there is limited but promising evidence for the safety and efficacy of MeVO EVT, and many neurointerventionists are already routinely offering EVT for MeVO stroke, despite the lack of clear guideline recommendations. In this article, we review the evidence on endovascular treatment for MeVO stroke and summarize the available literature on current imaging strategies, commonly used EVT selection criteria, EVT techniques, and outcome assessment for MeVO stroke.
\end{abstract}

In acute ischemic stroke, blockage of an intracranial artery leads to interruption of blood supply of the brain parenchyma with subsequent ischemia and infarction unless blood flow is restored quickly. Acute ischemic stroke has traditionally been divided into large-vessel occlusion (LVO) stroke, that is, acute stroke due to occlusion of proximal arteries of the anterior circulation (the terminal intracranial internal carotid artery (ICA), M1, or A1 segment), ${ }^{1}$ and non-LVO stroke. Around 35\%-40\% of acute ischemic stroke cases occur due to LVO, while $25 \%-40 \%$ are caused by medium-vessel occlusions (MeVOs). ${ }^{23}$ Although it is commonly assumed that outcomes of MeVO strokes are better compared with LVO strokes, due to the more distal occlusion location and less extensive ischemia, cohort studies suggest that outcomes are frequently poor, despite best medical management. ${ }^{4}$ Over the past few years, several randomized trials have proven the safety and efficacy of endovascular treatment (EVT) in LVO stroke, ${ }^{5}$ while for patients with MeVOs, there is currently no high-level evidence for EVT. But given the high efficacy of EVT in LVO stroke ${ }^{6}$ and the substantial morbidity associated with $\mathrm{MeVO},{ }^{4}$ EVT is now increasingly performed for MeVO stroke.

\section{DEFINITION OF MEDIUM-VESSEL OCCLUSIONS}

In a recent publication, MeVOs have been defined as occlusions of the M2, M3, A2, A3, P2, or P3 segment. ${ }^{2}$ That being said, the distinction between LVOs and MVOs is often challenging. For example, there are various ways to define the border between the M1 segment, which is considered a "large vessel", and the M2 segment ${ }^{1}$ of the middle cerebral artery. Furthermore, because M2 occlusions, particularly those affecting the dominant branch, can cause clinical symptoms identical to M1 occlusions, some authors include M2 occlusions in their definition of LVO. Given the variability in anatomy and clinical symptoms, a multidimensional definition based on morphological features (ie, vessel anatomy and size) and clinical deficits (ie, symptoms that are commensurate with the occluded vessel) may be preferred over a purely anatomical definition.

\section{PRIMARY AND SECONDARY MEVOS}

Not all MeVOs are the same: MeVOs can be classified based on their underlying mechanism as either primary or secondary MeVOs. "Primary" MeVOs occur de novo, with etiologies similar to LVOs. "Secondary" MeVOs occur when clot migration or fragmentation of a LVO occurs. ${ }^{8} 9$ This can happen spontaneously or iatrogenicly, after intravenous thrombolysis or during EVT. Secondary MeVOs may either represent embolization to new territory, if they are located outside the area that is affected by the primary occlusion, or embolization to distal territory, if they are located within the territory of the proximal occlusion. Another, rare subtype are "concurrent MeVOs", that is, MeVOs that occur simultaneously with other occlusions. They are likely part of an "embolic shower" or could be due to fragmentation of a more proximal occlusion.

\section{PREVALENCE AND CLINICAL COURSE OF MEVO STROKE}

Although it is commonly assumed that outcomes of MeVO strokes are better compared with LVO strokes, due to the more distal occlusion location and less extensive ischemia, a recent analysis from the INTERRSeCT and PRove-IT cohort studies has shown that one out of four patients with MeVO stroke does not achieve functional independence (modified Rankin Scale (mRS) 0-2) at 90 days with current best management, including intravenous thrombolysis if indicated. An excellent outcome (mRS 0-1) is only achieved by half of the patients with $\mathrm{MeVO}$ stroke (online supplemental figure 1). ${ }^{4}$ These somewhat sobering results emphasize the need for a more effective therapy. Given the overwhelming efficacy of endovascular treatment (EVT) in LVO strokes, it might seem obvious to expand this treatment to MeVO patients as well. Many neurointerventionalists already routinely offer EVT in MeVO strokes, particularly in the case of M2 
occlusions, ${ }^{710}$ but there is no randomized evidence for MeVO EVT, and it is possible that due to the smaller vessel size, MeVO EVT may yield an increased risk of procedural complications. ${ }^{11}$ Therefore, the question whether EVT is truly beneficial in the setting of MeVO stroke or not, remains to be answered. Furthermore, the smaller vessel caliber and more distal occlusion location of MeVOs compared with LVOs warrant changes in EVT technique and technology.

In the following, we review the existing literature on treatment indications, imaging selection criteria, and treatment techniques for MeVO EVT as well as clinical outcomes following MeVO EVT. We conclude with an outlook on open questions and ongoing developments.

\section{SEARCH STRATEGY}

We searched the electronic databases Medline/PubMed, Google Scholar, and EMBASE using the search terms stroke, thrombectomy, endovascular, aspiration, mechanical, small, distal, medium, M2, M3, A2, A3, P2, and P3 . We included original research studies, systematic reviews, and meta-analyses which: reported angiographic and/or clinical outcomes of patients with MeVO stroke treated with EVT; included at least five patients; and were published in English. Bibliographies of identified manuscripts were screened for additional relevant studies. The literature search is up-to-date as of 23 November 2020. Tables 1 and 2 provide an overview of the studies and systematic reviews that were identified during the literature search.

\section{IMAGING PROTOCOLS - HOW TO IMAGE MEVO STROKE?}

Baseline imaging can facilitate EVT treatment decision making in $\mathrm{MeVO}$ stroke, or enrolment in a randomized EVT MeVO trial for that matter, by answering the following questions:

1. Is there evidence of intracranial hemorrhage? Just as with LVO stroke, hemorrhagic stroke needs to be ruled out prior to treatment.

2. Is there an acute MeVO? EVT can only be initiated if a target occlusion is visualized.

3. Does that MeVO explain the patient's deficit (clinicalanatomical correlation, particularly important for secondary MeVOs and concurrent MeVOs in the setting of multiple occlusions)? Right-sided hemiparesis, for example, can be well-explained by a left-sided M2 occlusion, while left-sided hemi-paresis cannot.

4. Is the occlusion likely to recanalize with intravenous thrombolysis? Smaller, permeable thrombi may resolve with intravenous thrombolysis which may influence EVT decisionmaking.

5. Is the MeVO amenable to safe and fast recanalization using EVT? How technically challenging an operator perceives EVT for a certain MeVO and the availability of dedicated EVT tools designed for MeVOs will also influence the treatment decision and help to set the expectations of the patient and the medical team right.

Since EVT has been established as standard of care in $2015,{ }^{6}$ non-invasive neurovascular imaging (CTA or less frequently MR angiography) is recommended for all acute ischemic stroke patients, ${ }^{12}$ and should thus be obtained in all MeVO patients. In the past, detection of MeVOs on non-invasive imaging has been challenging, but innovative imaging techniques such as multiphase CTA (including color-coded mCTA maps) and CT perfusion have rendered fast and reliable MeVO detection possible $^{13-15}$ (online supplemental figure 2). Determining the presence of salvageable brain tissue is more challenging in MeVOs compared with LVOs. Established EVT imaging selection criteria that are currently used in LVO stroke, namely Alberta Stroke Program Early CT Score (ASPECTS) $\geq 6,{ }^{6}$ intermediate to good collaterals, ${ }^{16} 17$ and currently established perfusion mismatch/ core criteria (eg, ischemic core on rCBF maps) ${ }^{18-21}$ will likely be of little use in MeVO stroke. Due to the more distal occlusion, the ischemic territory will be smaller, and as such, ASPECTS will be generally higher and areas with severe hypoperfusion ("core") smaller. When assessing collateral status, one would have to assess collaterals solely within the affected territory downstream to the MeVO, which can be challenging, especially in the ACA and PCA territory. So far, no standardized methodology has yet been established. Due to the relative insensitivity and high interrater reliability of NCCT in detecting early ischemic changes, and because detecting MeVOs on conventional CTA/MRA images is difficult at times, advanced imaging protocols (CTP and MRI) are often used.

However, accurately determining infarct core and penumbra in MeVO stroke may be challenging or not possible at all, as recent literature suggests that we currently lack the ability to precisely delineate infarct "core" with routinely used imaging methods. $^{22}$ More importantly, delineating ischemic core and penumbra may not even be necessary, as past early time window LVO trials failed to show any volume cut-off below which EVT was no longer beneficial, and current EVT guidelines for LVO patients in the early time window therefore exclusively rely on NCCT ASPECTS to estimate the degree of irreversible tissue damage. ${ }^{6}$ That being said, using ASPECTS in MeVO stroke may be problematic, since the anterior and posterior cerebral artery territories are not represented at all, and even in middle cerebral artery MeVOs, the ASPECTS range will be relatively small, since the internal capsule, lentiform, and caudate are unlikely to be affected, and of the cortical 6 regions, only few will be affected. Thus, for a typical M3 occlusion, ASPECTS may be either 8, 9, or 10 .

Most of the MeVO EVT studies we identified used a CT-based imaging protocol, and approximately half of them included CT perfusion as part of the standard protocol, regardless of the time frame, and some reported MRI-based protocols with DWI \pm PWI sequences (table 2). Studies that relied only on NCCT and single-phase CTA were mostly subgroup analyses from randomized LVO trials and LVO registries, ${ }^{23}{ }^{24}$ that is, M2 occlusions were a small minority of all cases. Many studies simply transferred the perfusion thresholds ${ }^{25}$ and ASPECTS ${ }^{26}{ }^{27}$ cut-offs that are used for LVO strokes to their MeVO patient sample, which may be suboptimal for MeVO patient selection. Only a few studies adapted imaging selection criteria and used, for example, a higher ASPECTS cut-off. ${ }^{28}{ }^{29}$ Table 3 provides an overview of different $\mathrm{MeVO}$ imaging paradigms. Future studies should aim to develop MeVO-specific imaging selection criteria for EVT.

\section{CLINICAL EVT SELECTION CRITERIA FOR MEVO EVT}

Just as there are no uniform imaging criteria for EVT patient selection in MeVO strokes, there is also no consensus on clinical selection criteria. Several MeVO EVT studies used an NIHSS threshold of $\geq 6,{ }^{25-28}$ as it is currently recommended for LVO strokes. ${ }^{6}$ However, a recent analysis from prospective cohort studies has shown that $1 / 3$ of MeVO stroke patients have a baseline NIHSS $<6$. One study specifically stated that they included patients "with NIHSS $\geq 6$ or disabling deficit", 30 and another one lowered the NIHSS cut-off of 5 instead of $6,{ }^{29}$ which seems more appropriate, considering that one out of three patients with untreated supposedly "mild" strokes (NIHSS <5) will not be functionally independent at 90 days. ${ }^{31}$ Indeed, in a recent survey, most physicians stated that they would offer EVT for 


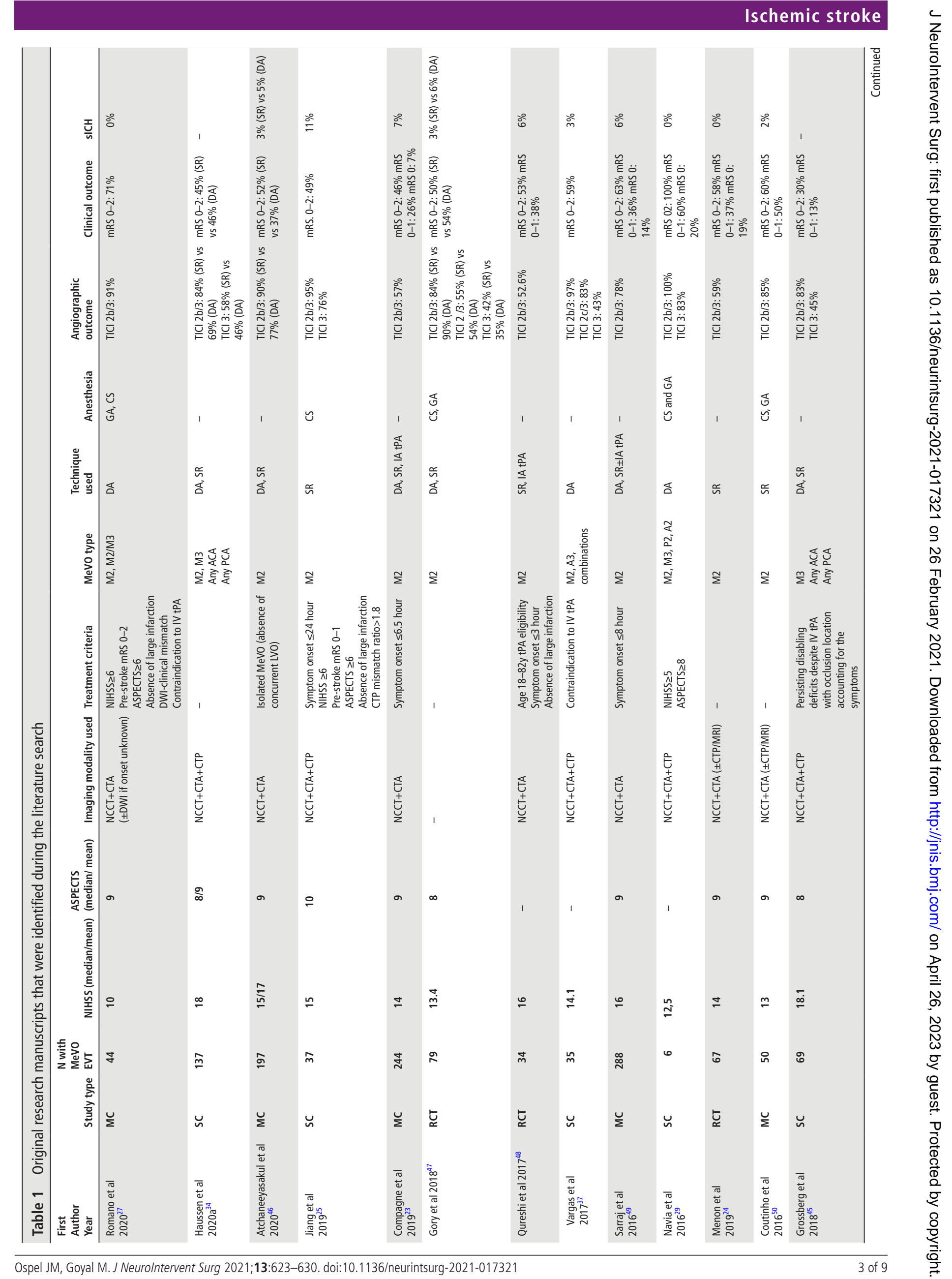




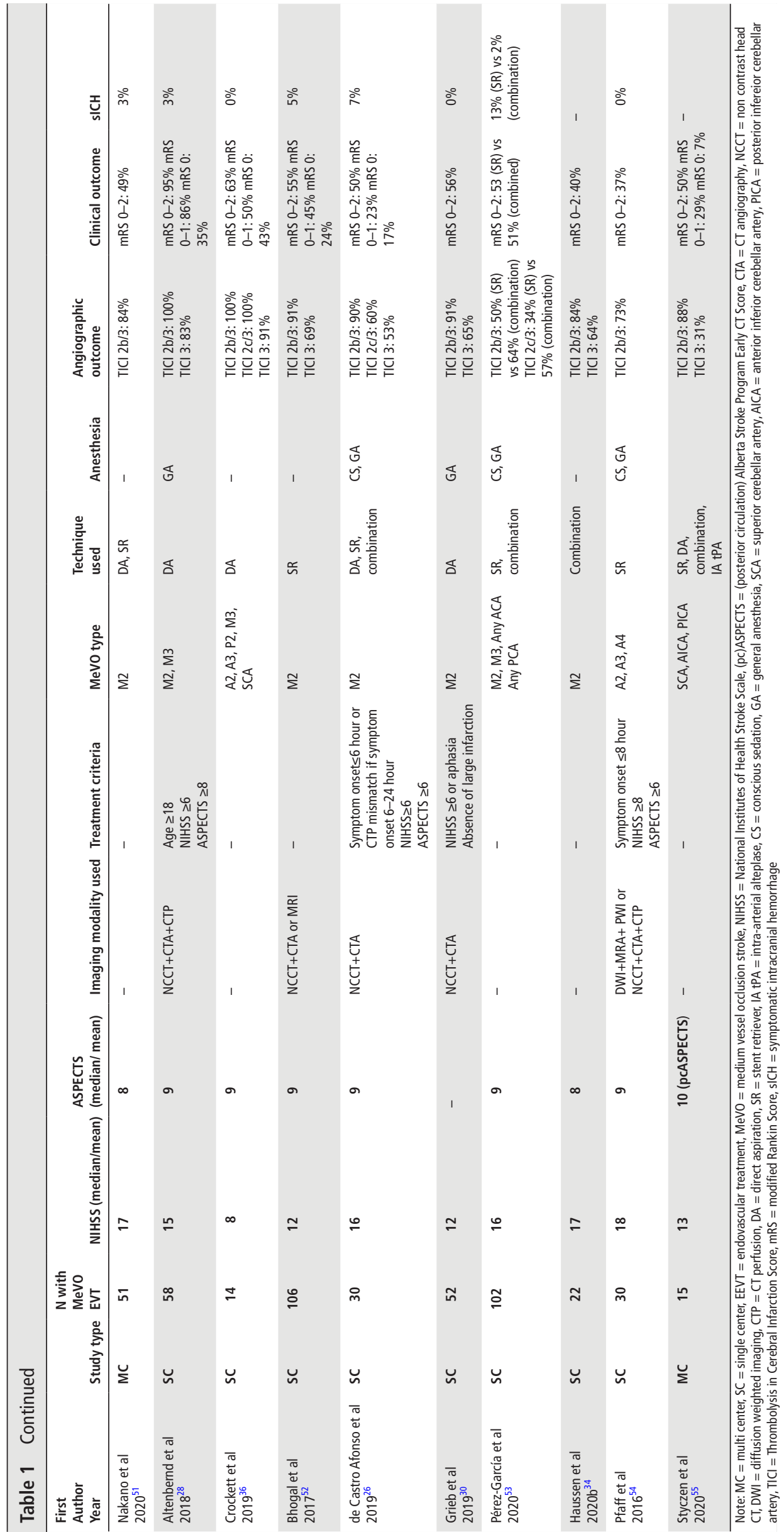


Table 2 Systematic reviews and meta-analyses that were identified during the literature search

\begin{tabular}{|c|c|c|c|c|c|c|c|c|c|c|c|c|}
\hline $\begin{array}{l}\text { First } \\
\text { Author } \\
\text { Year }\end{array}$ & $\begin{array}{l}\text { Study } \\
\text { type }\end{array}$ & Research question & Main findings & $\mathrm{N}$ of studies & $\begin{array}{l}\text { N with } \\
\text { MeVO } \\
\text { EVT }\end{array}$ & $\begin{array}{l}\text { NIHSS (median/ } \\
\text { mean) }\end{array}$ & $\begin{array}{l}\text { ASPECTS } \\
\text { (median/mean) }\end{array}$ & $\begin{array}{l}\text { MeVO } \\
\text { type }\end{array}$ & $\begin{array}{l}\text { Technique } \\
\text { used }\end{array}$ & $\begin{array}{l}\text { Angiographic } \\
\text { outcome }\end{array}$ & $\begin{array}{l}\text { Clinical } \\
\text { outcome }\end{array}$ & sICH \\
\hline $\begin{array}{l}\text { Phan et al } \\
2018^{38}\end{array}$ & MA & $\begin{array}{l}\text { What are the } \\
\text { reperfusion rates } \\
\text { in M2 occlusions } \\
\text { treated with SR } \\
\text { vs DA? }\end{array}$ & $\begin{array}{l}\text { Both SR and DA are } \\
\text { effective in recanalizing } \\
\text { M2 occlusions. The } \\
\text { literature is skewed by } \\
\text { DA being performed } \\
\text { sooner after onset of } \\
\text { stroke compared with } \\
\text { SR EVT. }\end{array}$ & 12 & 835 & 14 & 9 & $\begin{array}{l}\text { Mostly } \\
\text { M2 }\end{array}$ & $\begin{array}{l}\text { SR }(n=612) \text { vs } \\
\text { DA }(n=223)\end{array}$ & $\begin{array}{l}\mathrm{TICl} 2 \mathrm{~b} / 3: 81 \% \\
\text { (SR) vs } 87 \% \\
\text { (DA) } \\
\mathrm{TICl} 3: 54 \% \text { (SR) } \\
\text { vs } 57 \%(\mathrm{DA}) \\
\left(\mathrm{I}^{2}=37 \%\right)\end{array}$ & $\begin{array}{l}\text { mRS 0-2: } \\
60 \% \text { (SR) vs } \\
75 \% \text { (DA) } \\
\text { mRS 0- } 1: \\
40 \% \text { (SR) vs } \\
66 \%(D A) \\
\left(I^{2}=52 \%\right)\end{array}$ & $\begin{array}{l}6 \%(\mathrm{SR}) \text { vs } \\
3 \%(\mathrm{DA}) \\
\left(1^{2}=0 \%\right)\end{array}$ \\
\hline $\begin{array}{l}\text { Saber } \\
\text { et al } \\
2018^{42}\end{array}$ & MA & $\begin{array}{l}\text { What are the } \\
\text { angiographic results } \\
\text { and functional } \\
\text { outcome after M2 } \\
\text { EVT and how do } \\
\text { they compare to EVT } \\
\text { for LVO? }\end{array}$ & $\begin{array}{l}\text { M2 EVT is technically } \\
\text { feasible and safe } \\
\text { with high functional } \\
\text { independence. There } \\
\text { may be a slightly } \\
\text { increased risk of ICH. }\end{array}$ & 12 & 1080 & 14 & - & M2 & $S R, D A$ & $\begin{array}{l}\mathrm{TICl} 2 \mathrm{~b} / 3: 81 \% \\
\left(\mathrm{I}^{2}=20 \%\right)\end{array}$ & $\begin{array}{l}m R S 0-2: \\
59 \% \\
\left(1^{2}=61 \%\right)\end{array}$ & $\begin{array}{l}10 \% \\
\left(I^{2}=75 \%\right)\end{array}$ \\
\hline $\begin{array}{l}\text { Kim et al }{ }^{56} \\
2019\end{array}$ & MA & $\begin{array}{l}\text { What are the } \\
\text { angiographic results } \\
\text { and functional } \\
\text { outcome after M2 } \\
\text { EVT and how do } \\
\text { they compare to EVT } \\
\text { for M1 occlusions? }\end{array}$ & $\begin{array}{l}\text { M2 EVT is technically } \\
\text { feasible. Further studies } \\
\text { are needed to better } \\
\text { characterize the effect } \\
\text { of EVT in M2 occlusions. }\end{array}$ & 8 & 650 & - & - & M2 & - & $\begin{array}{l}\mathrm{TICl} 2 \mathrm{~b} / 3: 69 \% \\
\left(\mathrm{I}^{2} \text { not provided }\right)\end{array}$ & $\begin{array}{l}\text { mRS 0-2: } \\
59 \% \\
\left(I^{2} \text { not }\right. \\
\text { provided })\end{array}$ & $\begin{array}{l}6 \% \\
\left(I^{2} \text { not }\right. \\
\text { provided })\end{array}$ \\
\hline $\begin{array}{l}\text { Chen et } \\
\text { al }^{57} \\
2017\end{array}$ & SR & $\begin{array}{l}\text { What is the } \\
\text { evidence for M2 } \\
\text { EVT in the existing } \\
\text { literature? }\end{array}$ & $\begin{array}{l}\text { M2 EVT results in } \\
\text { high functional } \\
\text { independence with } \\
\text { modest ICH rates. But } \\
\text { given the relatively } \\
\text { favorable clinical } \\
\text { course of M2 occlusions } \\
\text { with conservative } \\
\text { management, the } \\
\text { benefits of EVT remain } \\
\text { unclear. }\end{array}$ & 8 & 630 & - & - & M2 & $S R, D A$ & $\begin{array}{l}\mathrm{TICl} 2 \mathrm{~b} / 3: 78 \% \\
\left(\mathrm{I}^{2} \text { not provided }\right)\end{array}$ & $\begin{array}{l}\mathrm{mRS} 0-2: \\
63 \% \mathrm{mRS} \\
0-1: 40 \% \\
\left(\mathrm{I}^{2} \text { not }\right. \\
\text { provided) }\end{array}$ & $\begin{array}{l}5 \% \\
\left(1^{2} \text { not }\right. \\
\text { provided })\end{array}$ \\
\hline
\end{tabular}

LVO patients with low NIHSS. ${ }^{32}$ Whether a patient is eligible for intravenous thrombolysis or not also seems to play an important role in MeVO EVT decision-making. More than 50\% of physicians would perform EVT in M3, A2, and P2 occlusions if the patient is ineligible for intravenous thrombolysis, but when intravenous thrombolytics can be safely administered, the willingness to proceed with EVT is substantially lower. ${ }^{7}$ For now, until randomized trial data become available, the decision to treat or not to treat a $\mathrm{MeVO}$ will remain a subjective one that is influenced by many factors, including patient preferences, eligibility for intravenous thrombolysis, and operator skills. Treatment decision-making is further complicated by the variety of clinical symptoms $\mathrm{MeVO}$ patients can present with, which are dependent on the eloquence of the affected area. ${ }^{3}$ A patient with a right-sided small branch anterior M2 occlusion, for example, may barely suffer from any deficits, but another patient suffering from a similar sized left-sided M2 occlusion may present with severe aphasia. Furthermore, new thrombolytic agents such as Tenecteplase, ${ }^{33}$ and neuroprotectants such as Nerinetide ${ }^{17}$ could improve the prognosis of $\mathrm{MeVO}$ stroke with conservative management and thereby also influence treatment decisionmaking in MeVO EVT.

\section{THROMBECTOMY TECHNIQUE - HOW TO PERFORM EVT IN MEVO STROKE?}

Several challenges emerge when performing EVT for MeVOs stroke. For the time being, we don't know how high the risk of vasospasm and dissection is when the catheter size matches or exceeds the vessel diameter, which can happen with largebore aspiration catheters in MeVOs. Medium-sized vessels can be too small to harbor a regular-sized distal access catheter (DAC), as most of the currently used DACs are 5-6F in diameter. Another problem is the DAC often gets stuck at the ledge of a bifurcation point such, such as the M1 bifurcation, but there are wedge-shaped microcatheters available to overcome this ledge effect. Furthermore, using a primary combined approach (ie, the combination of stent retriever, DAC, and balloon guide catheter), and advancing the system in a tri-axial manner, as it is commonly done for LVOs, may not be possible because of insufficient catheter length and diameter discrepancies. For example, using a longer DAC to access an M3 occlusion may not allow for a small enough and long enough microcatheter capable of deploying a stent. Thus, currently, the microwire and microcatheter are often introduced without a DAC. Once the microcatheter is in place, the stent can be deployed and the microcatheter removed before the distal access catheter is navigated to the site of occlusion (so-called "blind exchange mini-pinning technique"). ${ }^{34}{ }^{35}$ However, this is probably a transient problem, as many of the newer stent retrievers can be deployed through a 017 microcatheter and more and more microcatheters are now available in $160 \mathrm{~cm}$ length. On the other hand, numerous authors reported promising results of primary aspiration as the first-line approach in $\mathrm{MeVO}$ stroke, which may constitute an equally effective alternative to stent retriever-based techniques. ${ }^{27-30} 3637$ A comprehensive and unbiased comparison of different $\mathrm{MeVO}$ EVT techniques is currently not possible, since most studies are small, focus on specific devices, and a comparator group is often lacking. Furthermore, the literature on MeVO EVT techniques is biased, with onset-to-treatment times being on average much shorter in studies that used primary aspiration compared with those in which stent retrievers were used. ${ }^{38}$ Of note, MeVO EVT techniques and technologies are constantly evolving, and with MeVOs increasingly being targeted by neurointerventionalists, it is likely that treatment devices that are specifically tailored to MeVOs will be developed in the near future.

A substantial number of LVOs are treated without the use of general anesthesia (GA). Although randomized trials show equivalent or even better outcomes with GA vs conscious sedation, ${ }^{39}$ 


\begin{tabular}{|c|c|c|c|}
\hline $\begin{array}{l}\text { MeVO stroke imaging } \\
\text { protocols }\end{array}$ & Studies in which the protocol was used & Advantages & Disadvantages \\
\hline $\mathrm{NCCT}+\mathrm{CTA}$ & $\begin{array}{l}\text { Romano et al } 2020^{27} \text { (+DWI if symptom onset } \\
\text { unknown) } \\
\text { Atchaneeyasakul et al } 2020^{46} \\
\text { Compagne et al } 2019^{23} \\
\text { Qureshi et al } 2017^{48} \\
\text { Sarraj et al } 2016^{49} \\
\text { Menon et al } 2019^{24} \text { (CTP/MRI was available in } \\
\text { some patients) } \\
\text { Coutinho et al } 2016^{50} \\
\text { Bhogal et al } 2017^{52} \text { (either NCCT+CTA or MRI) } \\
\text { De Castro Afonso et al } 2019^{26} \\
\text { Grieb et al } 2019^{30}\end{array}$ & $\begin{array}{l}\text { Wide availability } \\
\text { Inexpensive } \\
\text { Fast } \\
\text { Robust against patient motion } \\
\text { No post-processing needed } \\
\text { Option to use multiphase CTA, including } \\
\text { color-coded time-variant mCTA maps and } \\
\text { mCTA-derived CTP-like maps }{ }^{1458}\end{array}$ & $\begin{array}{l}\text { If single-phase CTA is used MeVOs may be } \\
\text { missed }^{59} \\
\text { Little information about collateral status if } \\
\text { single-phase CTA is used } \\
\text { Current ASPECTS thresholds for LVO EVT are } \\
\text { probably not optimal for MeVO EVT } \\
\text { ASPECTS does not capture ischemic changes } \\
\text { in ACA and PCA MeVO stroke }\end{array}$ \\
\hline $\mathrm{NCCT}+\mathrm{CTA}+\mathrm{CTP}$ & $\begin{array}{l}\text { Haussen et al } 2020 a^{34} \\
\text { Jiang et al } 2019^{25} \\
\text { Vargas et al } 2017^{37} \\
\text { Navia et al } 2016^{29} \\
\text { Menon et al } 2019^{24} \text { (CTP/MRI was available in } \\
\text { some patients) } \\
\text { Coutinho et al } 2016^{50} \text { (CTP/MRI was available in } \\
\text { some patients) } \\
\text { Grossberg et al } 2018^{45} \\
\text { Altenbernd et al } 2018^{28} \\
\text { Pfaff } 2016^{54} \text { (either NCCT+CTA + CTP or } \\
\text { DWI+MRA + PWI) }\end{array}$ & $\begin{array}{l}\text { Higher information content compared with } \\
\text { NCCT+CTA only } \\
\text { Estimates for ischemic penumbra and } \\
\text { "core" volumes, also for ACA and PCA } \\
\text { MeVO stroke } \\
\text { Does not exclusively rely on ASPECTS for } \\
\text { ischemic "core" assessment } \\
\text { Option to use either single or multiphase } \\
\text { CTA }\end{array}$ & $\begin{array}{l}\text { Unvailability or limited availability of CTP in } \\
\text { smaller hospitals } \\
\text { Limited accuracy of "core" and penumbra } \\
\text { estimates due to variability in post- } \\
\text { processing mechanisms } \\
\text { - Susceptibility to patient motion }\end{array}$ \\
\hline MRI (DWI-MRI \pm PWI) & $\begin{array}{l}\text { Romano et al } 2020^{27} \text { (NCCT +CTA, DWI only if } \\
\text { symptom onset unknown) } \\
\text { Menon et al } 2019^{24} \text { (CTP/MRI was available in } \\
\text { some patients) } \\
\text { Bhogal et al } 2017^{52} \text { (either NCCT+CTA or MRI) } \\
\text { Pfaff et al } 2016^{54} \text { (either NCCT+CTA + CTP or } \\
\text { DWI+MRA + PWI) }\end{array}$ & $\begin{array}{l}\text { DWI: Highest sensitivity for acute small } \\
\text { infarcts } \\
\text { PWI: Estimates ischemic penumbra and } \\
\text { "core" volumes, also for ACA and PCA } \\
\text { MeVO stroke }\end{array}$ & $\begin{array}{l}\text { Limited availability of MRI and particularly } \\
\text { PWI in many hospitals } \\
\text { Contraindications } \\
\text { L Limited accuracy of PWI "core" and } \\
\text { penumbra estimates due to variability in } \\
\text { post-processing mechanisms } \\
\text { Susceptibility to patient motion } \\
\text { Various pitfallsin MRI interpretation due to } \\
\text { artifacts (eg, slow flow, metal artifacts due } \\
\text { to dental fillings, etc.) }\end{array}$ \\
\hline
\end{tabular}

$\mathrm{NCCT}=$ non contrast head CT, CTA = CT angiography, $C T P=C T$ perfusion, DWI = diffusion-weighted imaging, $\mathrm{MRA}=\mathrm{MR}$ angiography, $\mathrm{PWI}=$ perfusion-weighted imaging, $\mathrm{ACA}$ $=$ anterior cerebral artery, PCA = posterior cerebral artery, LVO = large vessel occlusion, ASPECTS = Alberta Stroke Program Eearly CT Score

data from the HERMES collaboration ${ }^{40}$ and individual EVT trials ${ }^{41}$ show worse outcomes with GA. It is possible that the use of GA will substantially increase when performing MeVO EVT, given the need for an excellent roadmap to get access to the relatively distally located clot. In addition, the anatomical variability is much higher in distal vessels. Sometimes, the MeVO may be right at the origin of a vessel branch, which requires the operator to blindly explore the site of the occlusion very gently in order to find the relevant vessel origin, which is much easier under GA, when patient movement is completely eliminated. Thus, the impact of an increased use of GA on functional outcome will need to be better understood.

\section{OUTCOME ASSESSMENT IN MEVO EVT}

\section{Clinical outcomes}

In $\mathrm{MeVO}$ strokes the area that is affected by ischemia is smaller than in LVO strokes. Thus, one would intuitively expect clinical outcomes to be better. Most studies that were identified in the literature search reported "good outcome", defined as mRS 0-2 at 90 days, as primary outcome (table 1). Given the overall better prognosis, it seems however worthwhile to consider a more restrictive outcome measure such as "excellent outcome", i.e. mRS $0-1$, or mRS shift analysis. Indeed, this has been recognized by several authors, who reported mRS $0-1$ or proportions of patients in each mRS category in addition to mRS $0-2$ (table 1 ).
It is important to note that deficits such as isolated abulia (due to A2/3 occlusion), alexia and agraphia (due to M2/3 occlusions), or quadrantanopia (due to P2/3 occlusion) are not well captured in the NIHSS and mRS. Not only are those scales limited in their granularity, they are also heavily focused on motor function and thus unable to capture the more subtle personality changes and domain-specific impairment that often play a dominant role in $\mathrm{MeVO}$ stroke-related disability.

\section{Angiographic outcomes}

In most MeVO EVT studies, angiographic outcomes are reported as "successful reperfusion", that is, TICI $2 \mathrm{~b} / 3$, analogous to reperfusion assessment in LVO stroke, although several studies reported TICI 3 and/or TICI $2 c / 3$ reperfusion in addition to TICI $2 \mathrm{~b} / 3$ rates (table 1 ). Reporting successful reperfusion (TICI $2 b / 3$ ) only is problematic, given that MeVOs in the anterior and posterior cerebral artery territory are not reflected in TICI at all, and patients with distal M2 or M3 occlusions will frequently have TICI $2 \mathrm{~b}$ at baseline. There is currently no satisfactory reperfusion grading system tailored to $\mathrm{MeVO}$ stroke. Crockett et al made an interesting attempt in this regard: in addition to the "regular" TICI, they used a modified TICI, which focused solely on the territory affected by the MeVO, with a scoring system identical to the standard TICI system $(1,2 \mathrm{a}, 2 \mathrm{~b}, 2 \mathrm{c}, 3) .^{36}$ Figure 1 shows a proposed modified eTICI scoring system for 


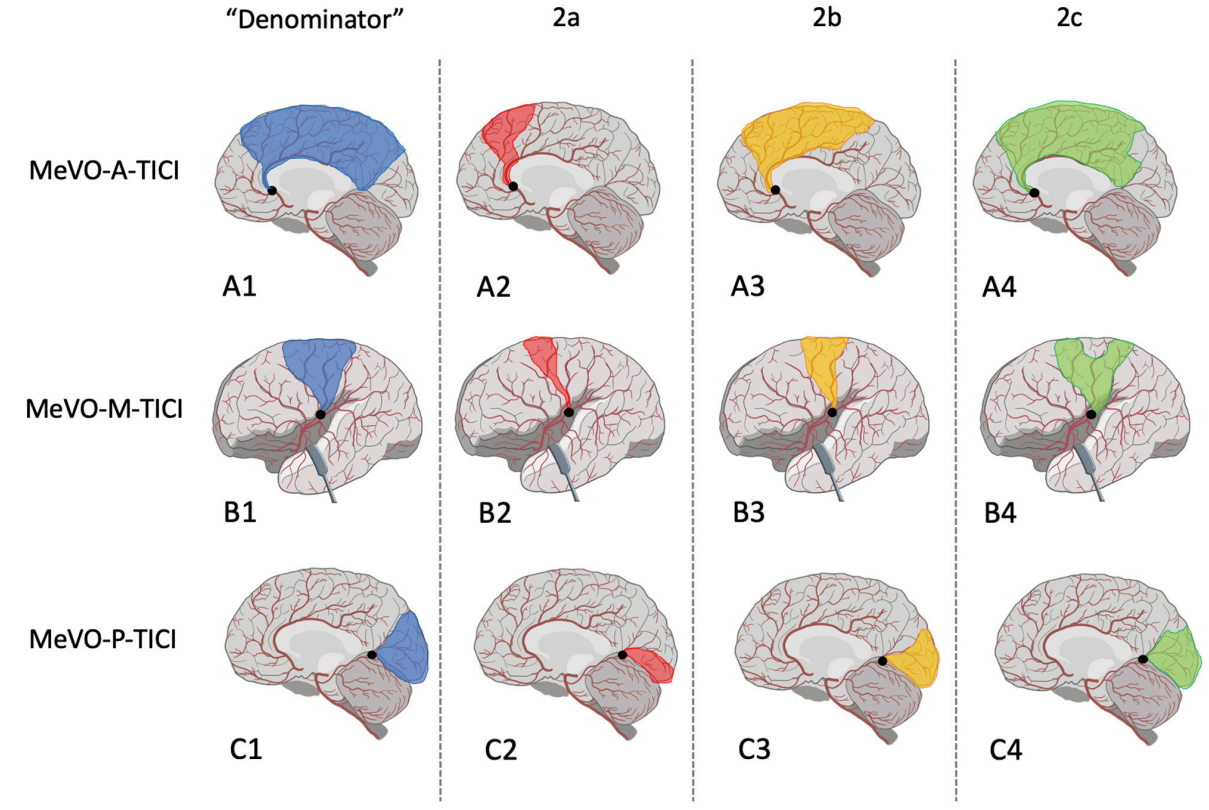

Figure 1 Suggested modified eTICl classification for MeVOs in the anterior (MeVO-A-TICl: A), middle (MeVO-M-TICl: B) and posterior (MeVO-P-TICl: C) cerebral artery. Blue overlays in (A1), (B1), and (C1) indicate the "denominator", that is, the territory downstream of the occlusion that serves as a comparator. Complete reperfusion of the territory marked in blue would correspond to an $\mathrm{ETICI}$ score of 3 . Reperfusion contained to territory marked with red overlays in (A2), (B2), and (C2) would indicate a MeVO-A-TICI, MeVO-M-TICl, and MeVO-P-TICI score of 2A, that is, reperfusion of less than $50 \%$ of the initially affected territory s. Reperfusion contained to territory marked with yellow overlays in (A3), (B3), and (C3) would indicate an MeVO-A-TICl, MeVO-M-TICl, and MeVO-P-TICl score of $2 \mathrm{~B}$, that is, reperfusion of $50 \%-90 \%$ of the initially affected territory . Reperfusion contained to territory marked with red overlays in (A4), (B4), and (C4) would indicate an MeVO-A-TICl, MeVO-M-TICl, and MeVO-P-TICl score of 2C, that is, nearcomplete reperfusion/ reperfusion of less than $90 \%-99 \%$ of the initially affected territory .

anterior, middle, and posterior cerebral artery MeVOs. The key difference to the conventional eTICI score is hereby the "denominator", that is, only the affected territory downstream to the $\mathrm{MeVO}$, rather than the entire middle cerebral artery territory, is used as a comparator.

\section{Safety outcomes}

Accurate and consistent reporting of safety outcomes is crucial when performing MeVO EVT, since the risk of complications is probably higher compared with LVOs, due to the relatively smaller vessel size and more distal occlusion location. Symptomatic intracranial hemorrhage $(\mathrm{sICH})$ was the most consistently reported complication type in MeVO EVT studies (table 1), although many reported asymptomatic hemorrhage, vasospasm, and extracranial complications as well. In two studies, sICH rates ranged from $10 \%-11 \%,{ }^{25} 42$ while the prevalence in other studies was below $8 \%$, which is only slightly higher compared with LVOs. ${ }^{5}$

\section{OUTCOMES FOR EVT IN PRIMARY VS. SECONDARY MEVOS}

Most of the literature published on MeVO EVT does not distinguish between primary and secondary MeVOs. But in particular the effect of "rescue" EVT in procedure-related secondary $\mathrm{MeVOs}$ is of great interest for neurointerventionalists, since peri-procedural embolization of clot fragments is a common phenomenon. Embolization causing anterior cerebral artery occlusion, for example, has been observed in more than $10 \%$ of LVO EVT procedures, ${ }^{43}$ especially in cases with terminal ICA occlusion, in which the clot segment extending into the ACA might get "guillotined off" by the distal aspiration catheter or stent retriever. ${ }^{44}$ Since the infarcted area will often be larger in secondary MeVOs, one would assume clinical outcomes to be worse compared with primary MeVO strokes. However, Grossberg et al, who reported post-EVT outcomes for primary and iatrogenic (EVT-induced) secondary MeVOs separately, found similar recanalization results and even slightly better clinical outcomes in patients with secondary MeVOs, despite more severe symptoms at baseline. ${ }^{45}$ Future studies on MeVO EVT should aim to capture information on MeVO types, and report results stratified for primary vs secondary MeVOs.

\section{CONCLUSION AND OUTLOOK}

With improving imaging technologies and a growing body of literature, MeVOs are being increasingly recognized as a target for EVT. Outcomes with intravenous thrombolysis, which is the current standard of care, are moderate at best and many patients are not eligible for thrombolytic treatment at all. At the present time, there is limited but promising evidence for the safety and efficacy of MeVO EVT. Many interventionists are already routinely treating primary as well as secondary MeVOs, that is, emboli into distal vessels during EVT for LVO stroke. However, standardized imaging protocols, treatment indication criteria, and unbiased comparisons of different EVT techniques for MeVOs are lacking. At the same time, imaging tools and particularly MeVO EVT techniques are rapidly evolving. A randomized controlled trial seems unavoidable in order to establish MeVO EVT as standard of care.

Twitter Johanna Maria Ospel @johanna_ospel and Mayank Goyal @mayank_G0

Acknowledgements The authors want to thank Basti Uhlmann for his help in designing the figure.

Contributors Both authors were involved in drafting and critical revisions of the manuscript. 
Funding The authors have not declared a specific grant for this research from any funding agency in the public, commercial, or not-for-profit sectors.

Competing interests MG is a consultant for Medtronic, Stryker, Microvention, GE Healthcare, Mentice.

Patient consent for publication Not required.

Provenance and peer review Commissioned; externally peer reviewed.

Supplemental material This content has been supplied by the author(s). It has not been vetted by BMJ Publishing Group Limited (BMJ) and may not have been peer-reviewed. Any opinions or recommendations discussed are solely those of the author(s) and are not endorsed by BMJ. BMJ disclaims all liability and responsibility arising from any reliance placed on the content. Where the content includes any translated material, BMJ does not warrant the accuracy and reliability of the translations (including but not limited to local regulations, clinical guidelines, terminology, drug names and drug dosages), and is not responsible for any error and/or omissions arising from translation and adaptation or otherwise.

\section{ORCID iDs}

Johanna Maria Ospel http://orcid.org/0000-0003-0029-6764

Mayank Goyal http://orcid.org/0000-0001-9060-2109

\section{REFERENCES}

1 Goyal M, Menon BK, Krings T, et al. What constitutes the M1 segment of the middle cerebral artery? J Neurointerv Surg 2016;8:1273-7.

2 Duloquin G, Graber M, Garnier L, et al. Incidence of acute ischemic stroke with visible arterial occlusion: a population-based study (Dijon Stroke Registry). Stroke 2020;51:2122-30

3 Saver JL, Chapot R, Agid R, et al. Thrombectomy for distal, medium vessel occlusions: a consensus statement on present knowledge and promising directions. Stroke 2020;51:2872-84

4 Ospel JM, Menon BK, Demchuk AM, et al. Clinical course of acute ischemic stroke due to medium vessel occlusion with and without intravenous alteplase treatment. Stroke 2020:51:STROKEAHA120030227.

5 Goyal M, Menon BK, van Zwam WH, et al. Endovascular thrombectomy after large-vessel ischaemic stroke: a meta-analysis of individual patient data from five randomised trials. Lancet 2016;387:1723-31.

6 Powers WJ, Rabinstein AA, Ackerson T, et al. Guidelines for the early management of patients with acute ischemic stroke: 2019 update to the 2018 guidelines for the early management of acute ischemic stroke: a guideline for healthcare professionals from the American Heart Association/American Stroke Association. Stroke 2019;50:e344-418.

7 Goyal M, Ospel JM, Menon BK, et al. MeVO: the next frontier? I Neurointerv Surg 2020;12:545-7.

8 Goyal M, Kappelhof M, McDonough R, et al. Secondary medium vessel occlusions: when clots move north. Stroke 2021:STROKEAHA120032799.

9 Ospel JM, Cimflova P, Marko M, et al. Prevalence and outcomes of medium vessel occlusions with discrepant infarct patterns. Stroke 2020;51:2817-24.

10 Almekhlafi M, Ospel JM, Saposnik G, et al. Endovascular treatment decisions in patients with M2 segment MCA occlusions. AJNR Am J Neuroradiol 2020;41:280-5.

11 Kurre W, Aguilar-Pérez M, Martinez-Moreno R, et al. Stent retriever thrombectomy of small caliber intracranial vessels using pREset LITE: safety and efficacy. Clin Neuroradiol 2017;27:351-60

12 Powers WJ, Rabinstein AA, Ackerson T, et al. 2018 guidelines for the early management of patients with acute ischemic stroke: a guideline for healthcare professionals from the American Heart Association/American Stroke Association. Stroke 2018;49:e46-110.

13 Menon BK, d'Esterre CD, Qazi EM, et al. Multiphase CT angiography: a new tool for the imaging triage of patients with acute ischemic stroke. Radiology 2015:275:510-20.

14 Ospel JM, Volny O, Qiu W, et al. Displaying multiphase CT angiography using a timevariant color map: practical considerations and potential applications in patients with acute stroke. AJNR Am J Neuroradiol 2020;41:200-5.

15 Yu AYX, Zerna C, Assis Z, et al. Multiphase CT angiography increases detection of anterior circulation intracranial occlusion. Neurology 2016:87:609-16.

16 Goyal M, Demchuk AM, Menon BK, et al. Randomized assessment of rapid endovascular treatment of ischemic stroke. N Eng/ J Med 2015:372:1019-30.

17 Hill MD, Goyal M, Menon BK, et al. Efficacy and safety of nerinetide for the treatment of acute ischaemic stroke (ESCAPE-NA1): a multicentre, double-blind, randomised controlled trial. Lancet 2020;395:878-87.

18 Albers GW, Marks MP, Kemp S, et al. Thrombectomy for stroke at 6 to 16 hours with selection by perfusion imaging. N Engl J Med 2018;378:708-18.

19 Campbell BCV, Mitchell PJ, Kleinig TJ, et al. Endovascular therapy for ischemic stroke with perfusion-imaging selection. N Engl J Med 2015;372:1009-18.

20 Saver JL, Goyal M, Bonafe A, et al. Stent-retriever thrombectomy after intravenous t-PA vs. t-PA alone in stroke. N Engl J Med 2015;372:2285-95.

21 Nogueira RG, Jadhav AP, Haussen DC, et al. Thrombectomy 6 to 24 hours after stroke with a mismatch between deficit and infarct. N Engl J Med 2018;378:11-21.
22 Goyal M, Ospel JM, Menon B, et al. Challenging the ischemic core concept in acute ischemic stroke imaging. Stroke 2020;51:3147-55.

23 Compagne KCJ, van der Sluijs PM, van den Wijngaard IR, et al. Endovascular treatment: the role of dominant caliber M2 segment occlusion in ischemic stroke. Stroke 2019;50:419-27.

24 Menon BK, Hill MD, Davalos A, et al. Efficacy of endovascular thrombectomy in patients with $\mathrm{M} 2$ segment middle cerebral artery occlusions: meta-analysis of data from the HERMES Collaboration. J Neurointerv Surg 2019:11:1065-9.

25 Jiang L, Xia W-Q, Huang H, et al. Mechanical thrombectomy outcome predictors in stroke patients with $\mathrm{M} 2$ occlusion: a single-center retrospective study. World Neurosurg 2019;127:e155-61.

26 de Castro Afonso LH, Borghini Pazuello G, Seizem Nakiri G, et al. Thrombectomy for M2 occlusions and the role of the dominant branch. Interv Neuroradiol 2019;25:697-704.

27 Romano DG, Frauenfelder G, Caragliano A, et al. Multicentric experience with an intermediate aspiration catheter for distal M2 ischemic stroke. J Stroke Cerebrovasc Dis 2020:29:105389.

28 Altenbernd J, Kuhnt O, Hennigs S, et al. Frontline ADAPT therapy to treat patients with symptomatic M2 and M3 occlusions in acute ischemic stroke: initial experience with the Penumbra ACE and 3MAX reperfusion system. J Neurointerv Surg 2018;10:434-9.

29 Navia P, Larrea J-A, Pardo E, et al. Initial experience using the 3MAX cerebral reperfusion catheter in the endovascular treatment of acute ischemic stroke of distal arteries. J Neurointerv Surg 2016;8:787-90.

30 Grieb D, Schlunz-Hendann M, Brinjikji W, et al. Mechanical thrombectomy of M2 occlusions with distal access catheters using ADAPT. I Neuroradiol 2019;46:231-7.

31 Spokoyny I, Raman R, Ernstrom K, et al. Defining mild stroke: outcomes analysis of treated and untreated mild stroke patients. J Stroke Cerebrovasc Dis 2015:24:1276-81.

32 Ospel JM, Kim B, Heo J-H, et al. Endovascular treatment decision-making in acute ischemic stroke patients with large vessel occlusion and low National Institutes of Health Stroke Scale: insights from UNMASK EVT, an international multidisciplinary survey. Neuroradiology 2020;62:715-21.

33 Campbell BCV, Mitchell PJ, Churilov L, et al. Tenecteplase versus alteplase before thrombectomy for ischemic stroke. N Engl J Med 2018;378:1573-82.

34 Haussen DC, Al-Bayati AR, Eby B, et al. Blind exchange with mini-pinning technique for distal occlusion thrombectomy. J Neurointerv Surg 2020;12:392-5.

35 Pérez-García C, Moreu M, Rosati S, et al. Mechanical thrombectomy in medium vessel occlusions: blind exchange with mini-pinning technique versus mini stent retriever alone. Stroke 2020:51:3224-31.

36 Crockett MT, Phillips TJ, Chiu AHY. Dual suction Headway27 microcatheter thrombectomy for the treatment of distal intracranial arterial occlusion strokes: initial experience with the micro-ADAPT technique. J Neurointerv Surg 2019:11:714-8

37 Vargas J, Spiotta AM, Fargen K, et al. Experience with a direct aspiration first pass technique (ADAPT) for thrombectomy in distal cerebral artery occlusions causing acute ischemic stroke. World Neurosurg 2017:99:31-6.

38 Phan K, Maingard J, Kok HK, et al. Contact aspiration versus stent-rRetriever thrombectomy for distal middle cerebral artery occlusions in acute ischemic stroke: meta-analysis. Neurointervention 2018;13:100-9.

39 Campbell D, Diprose WK, Deng C, et al. General anesthesia versus conscious sedation in endovascular thrombectomy for stroke: a meta-analysis of 4 randomized controlled trials. J Neurosurg Anesthesiol 2021;33:21-7.

40 Campbell BCV, van Zwam WH, Goyal M, et al. Effect of general anaesthesia on functional outcome in patients with anterior circulation ischaemic stroke having endovascular thrombectomy versus standard care: a meta-analysis of individual patient data. Lancet Neurol 2018;17:47-53.

41 Menon BK, Sajobi TT, Zhang Y, et al. Analysis of workflow and time to treatmen on thrombectomy outcome in the endovascular treatment for small core and proximal occlusion ischemic stroke (ESCAPE) randomized, controlled trial. Circulation 2016;133:2279-86.

42 Saber H, Narayanan S, Palla M, et al. Mechanical thrombectomy for acute ischemic stroke with occlusion of the M2 segment of the middle cerebral artery: a metaanalysis. J Neurointerv Surg 2018;10:620-4.

43 Kurre W, Vorlaender K, Aguilar-Pérez M, et al. Frequency and relevance of anterior cerebral artery embolism caused by mechanical thrombectomy of middle cerebral artery occlusion. AJNR Am J Neuroradiol 2013;34:1606-11.

44 McTaggart RA, Ospel JM, Psychogios M-N, et al. Optimization of endovascular therapy in the neuroangiography suite to achieve fast and complete (expanded treatment in cerebral ischemia 2c-3) reperfusion. Stroke 2020;51:1961-8.

45 Grossberg JA, Rebello LC, Haussen DC, et al. Beyond large vessel occlusion strokes: distal occlusion thrombectomy. Stroke 2018:49:1662-8.

46 Atchaneeyasakul K, Malik AM, Yavagal DR, et al. Thrombectomy outcomes in acute ischemic stroke due to middle cerebral artery M2 occlusion with stent retriever versus aspiration: a multicenter experience. Interv Neurol 2020:8:180-6.

47 Gory B, Lapergue B, Blanc R, et al. Contact aspiration versus stent retriever in patients with acute ischemic stroke with $\mathrm{M} 2$ occlusion in the aster randomized trial (contact aspiration versus stent retriever for successful revascularization). Stroke 2018;49:461-4. 
48 Qureshi Al, Saleem MA, Aytac E. Comparison of endovascular treatment with intravenous thrombolysis for isolated $\mathrm{M} 2$ segment of middle cerebral artery occlusion in acute ischemic stroke. J Vasc Interv Neurol 2017;9:8-14.

49 Sarraj A, Sangha N, Hussain MS, et al. Endovascular therapy for acute ischemic stroke with occlusion of the middle cerebral artery M2 segment. JAMA Neurol 2016;73:1291-6.

50 Coutinho JM, Liebeskind DS, Slater L-A, et al. Mechanical thrombectomy for isolated M2 occlusions: a post hoc analysis of the StAR, swift, and swift prime studies. AJNR Am J Neuroradiol 2016;37:667-72.

51 Nakano T, Shigeta K, Ota T, et al. Efficacy and Safety of Mechanical Thrombectomy for Occlusion of the Second Segment of the Middle Cerebral Artery : Retrospective Analysis of the Tama-REgistry of Acute endovascular Thrombectomy (TREAT). Clin Neuroradiol 2020;30:481-7.

52 Bhogal P, Bücke P, Aguilar Pérez M, et al. Mechanical thrombectomy for M2 occlusions: a single-centre experience. Interv Neurol 2017;6:117-25.

53 Pérez-García C, Moreu M, Rosati S, et al. Mechanical thrombectomy in medium vesse occlusions: blind exchange with Mini-Pinning technique versus mini stent retriever alone. Stroke 2020;51:3224-31.
54 Pfaff J, Herweh C, Pham M, et al. Mechanical thrombectomy of distal occlusions in the anterior cerebral artery: recanalization rates, periprocedural complications, and clinical outcome. AJNR Am J Neuroradiol 2016;37:673-8.

55 Styczen H, Fischer S, Yeo LL, et al. Approaching the Boundaries of Endovascular Treatment in Acute Ischemic Stroke : Multicenter Experience with Mechanical Thrombectomy in Vertebrobasilar Artery Branch Occlusions. Clin Neuroradiol 2020. doi:10.1007/s00062-020-00970-7. [Epub ahead of print: 27 Oct 2020].

$56 \mathrm{Kim} \mathrm{CH}$, Kim S-E, Jeon JP. Meta-Analysis of endovascular treatment for acute M2 occlusion. J Korean Neurosurg Soc 2019;62:193-200.

57 Chen C-J, Wang C, Buell TJ, et al. Endovascular mechanical thrombectomy for acute middle cerebral artery M2 segment occlusion: a systematic review. World Neurosurg 2017;107:684-91.

58 Ospel JM, Qiu W, Goyal M. Missed medium-vessel occlusions on CT angiography: make it easier ... easily! AJNR Am J Neuroradiol 2020;41:E73-4.

59 Fasen BACM, Heijboer RJJ, Hulsmans F-JH, et al. CT angiography in evaluating large-vessel occlusion in acute anterior circulation ischemic stroke: factors associated with diagnostic error in clinical practice. AJNR Am J Neuroradiol 2020;41:607-11. 